\title{
Evaluation of Enterprise Performance Based on FLI-GA Model
}

\author{
Shouyi Zhang ${ }^{1}$, Xiaomin Zhu ${ }^{1}$, Jiandong Geng ${ }^{2}$, Runtong Zhang ${ }^{3 *}$ \\ ${ }^{1}$ School of Mechanical, Electronic and Control Engineering, Beijing Jiaotong University, \\ ${ }^{2}$ The High Technology Research and Development Center, The Ministry of Science and Technology of the People's \\ Republic of China, \\ ${ }^{3}$ School of Economics and Management, Beijing Jiaotong University (China) \\ shouyizhang@126.com,xmzhu@,bjtu.edu.cn,gjd5918@163.com,rtzhang@bjtu.edu.cn*Corresponding author
}

\section{Abstract:}

Purpose: There are many kinds of methods to evaluate the performance of enterprise, but they still have some distinct shortcomings. In order to achieve a better evaluation result, we put forward a new model named FLI-GA (Fuzzy logic inference \& Genetic algorithm).

Design/methodology: This model, mainly based on the fuzzy logic inference method, uses fuzzy rule-based system (FRBS) to avoid the drawbacks of FRBS. Genetic algorithm is applied in this model.

Findings: FLI-GA model can be used to evaluate certain enterprise performance, and its evaluation results are more accurate than fuzzy logic inference method.

Originality/value: This model combines the genetic algorithm with the unclear reasoning methods so as to make the appraisal results more reasonable and more satisfying.

Keywords: FLI-GA model, performance evaluation, genetic algorithm 


\section{Introduction}

Evaluating the enterprise management performance in a scientific way is helpful to obtain accurate guidance of enterprise management behaviors. This evaluation is essential to improve the management methods of enterprises. Besides, this kind of evaluation helps to strengthen the innovation of enterprise management system, as well as to enhance the achievement inspection efficiency of the operators. Such evaluation can assist the establishment of the encouragement and restraint mechanism. On one hand, it is conductive to the enhancement of the image consciousness and competitive power of certain enterprises. On the other hand, simultaneously, it also provides the basis for the macroeconomic regulation and the formulation of the economic policies as well. Therefore the evaluating performance of enterprise is playing a more and more vital role in modern enterprises.

\subsection{Brief Definition of Enterprise Performance Evaluation}

Enterprise Performance evaluation can make objective and fair judgment of an enterprise's operational effectiveness in a certain operating time. This will help to guide this enterprise to promote its reform and innovation, thereby enhance the competitiveness of this enterprise. Modern enterprises cannot neglect the important role played by performance evaluation.

As a multi-index comprehensive evaluation, the popular enterprise performance evaluation methods mainly include Analytical Hierarchy Process (AHP), artificial neural network (ANN) evaluation method, grey correlation method, fuzzy comprehensive evaluation method, genetic algorithm, factor analysis, TOPSIS and so on.

\subsection{Literature Review of Enterprise Performance Evaluation}

After the born of modern enterprises, ownership rights was separated from management rights. In order to protect their own interests, owners of capital generally assess operators' subjective effort and their operating results. Over the years, a variety of evaluation thinking conceived and became more ripe and satisfactory. There are 3 comprehensive evaluation methods from the view of corporate finance:

- Dupont Financial Analysis System. This system attaches great importance to integrity of management and operations, which also outstands the coordination and multi-layer of management.

- Wall Marking Way. Irrelevant financial indexes are assigned with different proportion, making it possible to comprehensively calculate the financial situation of enterprises. 
The deficiencies of this method are unreasonable score calculation method and inadequate indexes' proof.

- EVA Evaluation System. EVA indexes overcome the traditional profit indexes' ignorance that the value of corporate assets will change over time, as well as the opportunity cost of owner's equity. Although it has a great progress, EVA evaluation system still has limitations, such as financial-oriented and short-term-oriented.

As is shown in the former section, there are also some popular methods and their improved models.

The advantages of AHP method (Golam \& Akhtar, 2011; Bi \& Zhang, 2010) is its extremely precise value, higher scientific basis. The more obvious shortcomings are its poor operability, the more artificial color when determine the relative weights, and less intuitive. The neural network method has strong robustness, memory capability, nonlinear mapping ability and a strong self-learning ability, but has no ability to explain its reasoning process and reasoning basis.

Artificial neural network (Lou \& Kuang, 2010) has self-learning function, association and storage capabilities and high-speed ability to find the optimal solution. But its architecture is not versatile, and it is difficult to accurately analyze the performance of the neural network.

The gray relational analysis (Yang, Zhang, Wang, Wang \& Zhang, 2010) requirements are lower and have less calculation. But the process of computing weights has poor objectivity, which does not meet some of the more important samples.

Fuzzy comprehensive evaluation (Ma, He \& Shuai, 2011) method's results are clear and have systematic characteristics. This method can solve the problems of fuzzy and difficult to quantify, so it is suitable for a variety of non-deterministic problems. The disadvantages of this method are its duplication of information, subjectivity, and the difficulty of determine the membership function.

Genetic algorithm (Iraj, Mahyar \& Fereydoun, 2011) has good convergence in the calculation of accuracy requirement, less computation time, high robustness. By contrast, the drawbacks are that sometimes it may be precocious and needs large amount of computation. Besides, it has poor stability and small data scale.

Factor analysis (Patrick, Douglas \& Fabio, 2010) can use computer software to solve problems quickly and easily. Therefore, compared with other methods, factor analysis is a scientific, practical, simple and comprehensive evaluation method. However, Factor analysis is just a kind of financial analysis method which is only on the basis of financial indicators, macroeconomic factors and some special cases. 
TOPSIS (Saeed, Mehdi \& Mostafa, 2012) method is an ideal target sequence optimal selection technology; it's a very effective method in multi-objective decision. But the drawback of subjective weights and reverse problem limit its application.

\section{Fuzzy Logic Inference Method and its Shortage}

\subsection{Introduction of Fuzzy Logic Inference Method}

In the process of evaluating the enterprise performance, the subjective factor of human is uncertain, which makes it difficult to compare the values of different indexes. Even the experts in this domain can hardly give exact weight of each individual index. Moreover, weight is dynamic and alterable, so the value of index's weight maybe improbable. How to deal with this problem? The answer is fuzzy logic inference method (Rustum, Omar \& Emad, 2009). Fuzzy logic inference method can reflect the expert's correct judgment, so as to avoid difficulties of giving weights directly; in addition, as long as the satisfaction of the membership function (fuzzy inference rules) level is more than enough, experts' judgment can approximate the actual situation.

On account of the above advantages, our new model will be improved basing on fuzzy inference method. The fuzzy logic inference method uses fuzzy rule-based system (shortened form FRBS). It allows assessors to divide a complex system into parts in order to easily handle this system. FRBS can also use the data of various types (qualitative or quantitative data) and it allows a small amount of measurement error.

\subsection{Evaluation Steps of Fuzzy Logic Inference Method}

After the analysis of former section, we can refine the reasoning steps of the fuzzy inference method. The evaluation process of fuzzy logic inference method contains 7 steps:

- First substitute the indexes scores into the predetermined membership function, and then get membership of each index (the fuzzy set 1) through membership function.

- Memberships are compared with the corresponding rule base. The minimum principle should be adopted during above process.

- Use the maximum principle to contrast results in order to screen better membership after the comparison within the rule base, and thus get the memberships of the middlelevel evaluation indexes-fuzzy sets2.

- Fuzzy set 2 is compared with related rule base. Then we repeat step 2 . 
- We use the same method as step 3 to get the membership degree of the high tier evaluation indexes-fuzzy sets3.

- The fuzzy sets of major and secondary factor are both substituted into the rule base to compare the two fuzzy sets with their related rule bases. The same maximum and minimum principles are applied as previous steps. At last, the final evaluated fuzzy sets are given out.

- Compare the degree of fuzzy sets' memberships which vary from well, ordinary to bad. According to the maximum principle of membership, the final analysis conclusion is obtained.

\subsection{Shortage of Fuzzy Logic Inference Method}

Fuzzy logic inference method introduced above first uses triangular membership function in fuzzy mathematics to calculate the membership of the underlying index, and then use the rule base layers to get new membership values of upper tier's indexes. This method makes the calculus process inevitably rigid, thus the result is singleness to the rate for the same group, so that it is not conducive to the latter part of the analysis and further assessment work.

\section{Building the FLI-GA model}

We propose an improved model based on the fuzzy logic inference method and genetic algorithm method-FLI-GA model (Fuzzy Logic Inference \& Genetic Algorithm Model). After calculate the lowest tier indexes' values, the improved FLI-GA model makes use of genetic algorithms to generate offspring and choose the best individual. The calculation process is relatively flexible and easy to understand, and its multiple results obtained from repeated computing can be applied for post- analysis and selection.

\subsection{Brief introduction of Genetic Algorithm}

Genetic Algorithm (GA) (Pond, Posada, Gravenor, Woelk \& Frost, 2006) is a class of random search method. It learns from biological laws of evolution (survival of the fittest) and is evolved from this law. It was first proposed by Professor J. Holland from the United States in 1975. GA's main feature is that it directly operates the objects and there are no limits about derivation and function continuity. GA has inherently implicit parallelism and better ability of global optimization. Besides, its probabilistic optimization method can automatically access and guide the optimal search space. It can also adaptively adjust the search direction, and need 
not to decide the rules. These properties of GA have been widely used in combination optimization, machine learning, signal processing, adaptive control, artificial life and other important fields.

\subsection{Build index system of FLI-GA model}

The relate studies of enterprise performance evaluations (Zhang, Xie, Cao, 2011; Zhang \& Tan, 2012; Qi \& Sun, 2011) are only focus on the cost information and financial information. This kind of models ignores the quality and sense of time; neither do the non-financial information like human capital and so on. To avoid the drawbacks of traditional enterprise performance evaluation, new research achievements are included in our study. By considering the normal principle of index selection and characteristics of enterprise performance evaluation, we initially screen 13 indexes from the views of financial, business growth ability, internal management and workforce capability. The indexes are shown in Table 1.

\begin{tabular}{|c|c|c|c|}
\hline Type & Num. & Index & Measurement \\
\hline \multirow{4}{*}{$\begin{array}{l}\text { Assets } \\
\text { operation } \\
\text { situation }\end{array}$} & 1 & Turnover of current assets & $\begin{array}{l}\text { A unit's current assets turnover time } \\
\text { per unit time }\end{array}$ \\
\hline & 2 & Total Assets Turnover & Total asset's turnover time per unit time \\
\hline & 3 & Interest earned multiple & $\begin{array}{l}\text { (Relative to the start-up capital) } \\
\text { Multiple of earnings }\end{array}$ \\
\hline & 4 & Asset-liability ratio & (Total liabilities/Total assets) $\times 100 \%$ \\
\hline \multirow{4}{*}{$\begin{array}{l}\text { Growth ability } \\
\text { condition }\end{array}$} & 5 & Marketing Ability & $\begin{array}{l}\text { Enterprise's market adaptation and } \\
\text { control ability }\end{array}$ \\
\hline & 6 & Operating income growth & The growth rate of operating income \\
\hline & 7 & Corporate culture & $\begin{array}{l}\text { Background and culture included in } \\
\text { growth process }\end{array}$ \\
\hline & 8 & Corporate Recognition & Social awareness of an enterprise \\
\hline \multirow{3}{*}{$\begin{array}{l}\text { Employee } \\
\text { productivity }\end{array}$} & 9 & Employees' enthusiasm & The enthusiasm of the employees \\
\hline & 10 & Employee learning ability & $\begin{array}{l}\text { Ability of accepting and learning new } \\
\text { things }\end{array}$ \\
\hline & 11 & Staff training & $\begin{array}{l}\text { Manufacturing capacity of the excellent } \\
\text { staff }\end{array}$ \\
\hline \multirow[b]{2}{*}{$\begin{array}{l}\text { Organization } \\
\text { policy }\end{array}$} & 12 & The company departments & Distinguish between degrees of work \\
\hline & 13 & Company staff redundant & $\begin{array}{l}\text { Whether the human resource } \\
\text { deployment is rational }\end{array}$ \\
\hline
\end{tabular}

Table 1. Primary indexes of enterprise performance evaluation

The final index system is built after the initial indexes were selected. Some standards are implemented when select the final indexes:

- The selected indexes should reflect two factors: absolute and relative number;

- The selected indexes should be simple, clear and practicable;

- It is necessary to select the quantitative as well as qualitative indexes; 
- Current corporate outcome indexes and strategic targets should be both considered.

Based on the principles above and the indexes in Table 1, a new index system is screened out, which is shown in Figure 1.

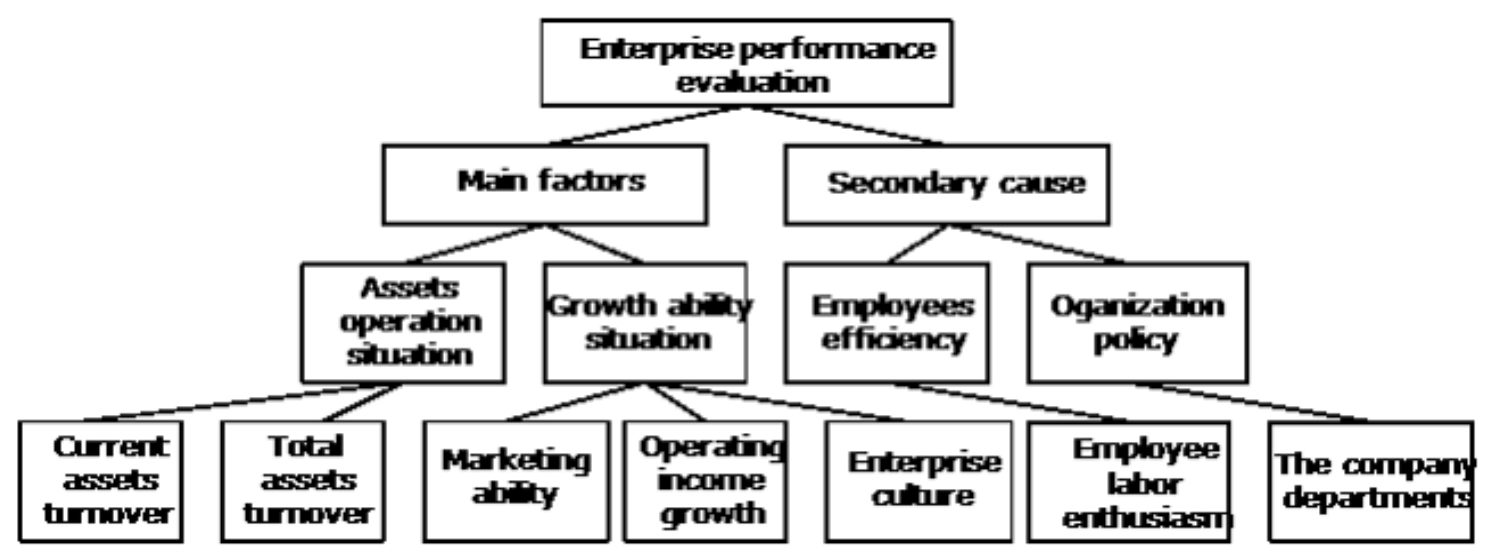

Figure 1. Index system after screening

To clarify this further, the final index system will be divided into four parts to be explained:

- Asset operational status: whether a company is profitable or not is the key factor of enterprise performance. Operation of assets mainly includes current assets turnover and total assets turnover status.

- Business growth ability of an enterprise decides its fate. Marketing ability relates to the enterprise market share and product awareness. Good corporate culture helps to establish a good public image and corporate reputation, and gives great incentives to subordinates.

- Cumulative employee productivity is just the overall efficiency of an enterprise.

- The organizational policy of an enterprise influences the enterprise's internal resources configuration.

Strictly speaking, every enterprise should have a very individualized performance evaluation system. This index system is designed on the basement of the commonality of general enterprises. In the practical application, evaluation system should be appropriately changed according to the enterprise that is evaluated. 


\subsection{The algorithm of FLI-GA model}

The algorithm of FLI-GA model will be introduced after building the evaluation index system.

\subsubsection{The determination of the lowest tier indexes' membership functions}

There are three common methods (subjective experience method, analytical reasoning method, survey $\&$ statistics method) to determine the membership function.

According to the nature of the problem, analytical reasoning method applies some definite analyses and reasoning method to select typical function as membership function in the continuous domain. Furthermore, several principles should be considered when selecting the membership function: Membership functions should be simple; Meet the convex fuzzy set principles; Select less evaluation indexes and rules to reduce complex calculations; Try to satisfy the requirement of non-overlapping membership; Describe the transition relations intuitively among the standard values from different reviews.

So according to these regulations, triangular membership function is selected as it fits the experience and principles above. We respectively select $a_{1} 、 a_{2} 、 a_{3}$ as $0,2.5,5$ (the index variation range is $[0,5]$ ) and get triangular membership function of the bottom indexes of FLIGA model.

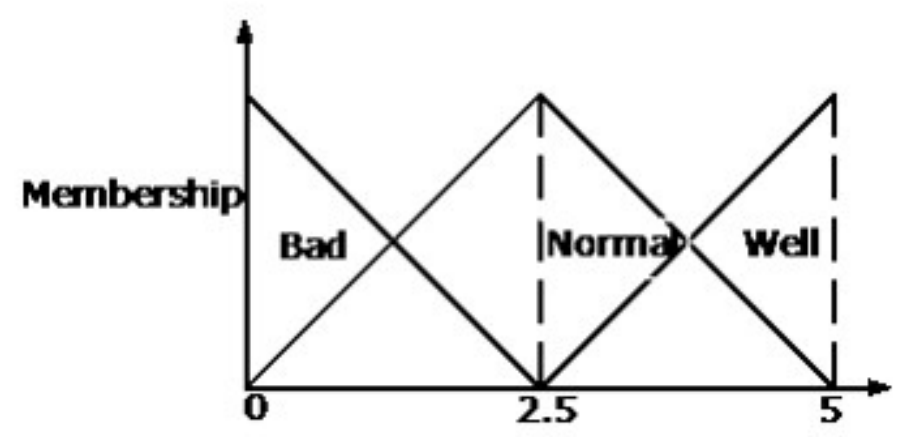

Figure 2. Membership function of bottom index

The membership function is demonstrated as follows:

$$
A_{1}(x)=\left\{\begin{array}{cc}
\frac{2.5-x}{2.5} & 0 \leq x \leq 2.5 \\
0 & 2.5 \leq x \leq 5
\end{array}\right.
$$




$$
\begin{gathered}
A_{2}(x)=\left\{\begin{array}{cc}
\frac{x}{2.5} & 0 \leq x \leq 2.5 \\
\frac{5-x}{2.5} & 2.5 \leq x \leq 5
\end{array}\right. \\
A_{J}(x)=\left\{\begin{array}{cc}
0 & 0 \leq x \leq 2.5 \\
\frac{x-2.5}{2.5} & 2.5 \leq x \leq 5
\end{array}\right.
\end{gathered}
$$

After the establishment of triangular membership function, we built the regulation base for model application according to the expert experience.

Bottom tier reasoning rules will be applied to get indexes' reasoning output of the Fuzzy logic inference model and improved FLI-GA model. The rule base of the upper index applies only to the upper index of the Fuzzy logic inference model, while FLI-GA model uses improved reasoning mechanism based on genetic algorithm. The reasoning rule base of the upper indexes will be applied in the next section to realize the simulation comparison between the two models.

\subsubsection{Heredity and variation of the upper indexes and their individual fitness}

Based on Genetic Algorithms, we select three membership values from bottom tier as the male parent of the three fitness: fitness ${ }_{b a d}$, fitness normal, $_{\text {fitness }}$ well. Their membership values are the same and fitness values vary from 0 to 1 . Particularly, when one of the male parent' value is zero, we choose another paternal fitness value as the corresponding fitness value of its offspring. We marked the selected couple of male parents as $A_{1}$ and $A_{2}$. During the first genetic round, their corresponding fitness values were set as fitness $s_{1}(t)$, fitness $s_{2}(t)$. So we could calculate the probability of the selected allele:

$$
\begin{aligned}
& p_{1}=\text { fitness }_{1}(t) /\left[\text { fitness }_{1}(t)+\text { fitness }_{2}(t)\right] \\
& p_{2}=\text { fitness }_{1}(t) /\left[\text { fitness }_{1}(t)+\text { fitness }_{2}(t)\right]
\end{aligned}
$$

Then we selected allele through $A_{1}$ or $A_{2}$ according to $p_{1}$ and $p_{2}$, the specific operation is explained as follows: Set $i=1,2,3 \ldots L$ ( $L$ is the string length of the individual). Firstly, generate a sequence $a_{i}(i=1,2,3 \ldots)$ which varies from 0 to 1 . Then compare a1 with $p_{1}$ or $p_{2}$ to determine the selected allele. Here, one should notice that when $p_{1} \leq p_{2}$, if $a_{1} \leq p 1$ then select 
the allele of $A_{1}$, else if when $a_{1}>p_{1}$ then select the allele of $A_{2}$, and while $p_{1}>p_{2}$, if $a_{1} \leq p_{2}$ then select the allele of $A_{2}$, else if $a_{1}>p_{2}$, then select the allele of $A 1$. After the former 2 steps, repeat step 2, until a daughter chromosome is produced.

\subsubsection{Choose the best for the daughter chromosome}

Due to the gene mutations, a population may have the "super individual" and "ultra-small individual" during the process of inheritance. In this case, fitness value maybe too high or too low.

In order to achieve more reliable and reasonable evaluation results, we made a couple of male parents mate for 500 times in the FLI-GA model. Then we took the first 100 values of the corresponding indexes' fitness into averaging operation, the resulting value is the upper index corresponding fitness.

\subsubsection{Non-fuzzy processing of indexes}

The final enterprise performance evaluation has three fitness values. Three fitness values were compared among the three "poor", "normal" and "well" memberships. The max fitness value is the enterprise's performance evaluation rating. In this example, we simply adopted three evaluation levels - "poor", "normal" and "well".. In the practical application, evaluation grade can be expanded, such as being expanded as follows: "good", "better", "normal", "poor", "bad" five levels, it will give a more detailed result. In a word, the FLI-GA model is just playing a valuable role named "throw a sprat to catch a whale" in this article. The actual use of enterprise performance evaluation needs continuous studying and improving.

The whole process of building FLI-GA model can be summarized as the following 3 figures. (Figures 3-5). 


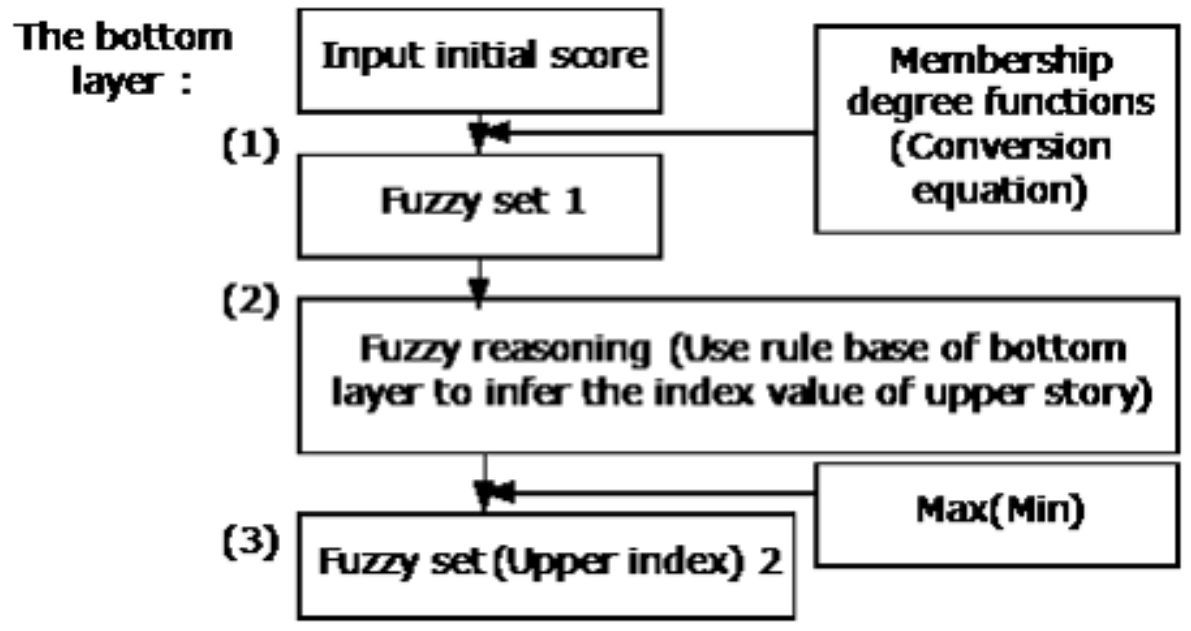

Figure 3. Computing the bottom index value of FLI-GA model

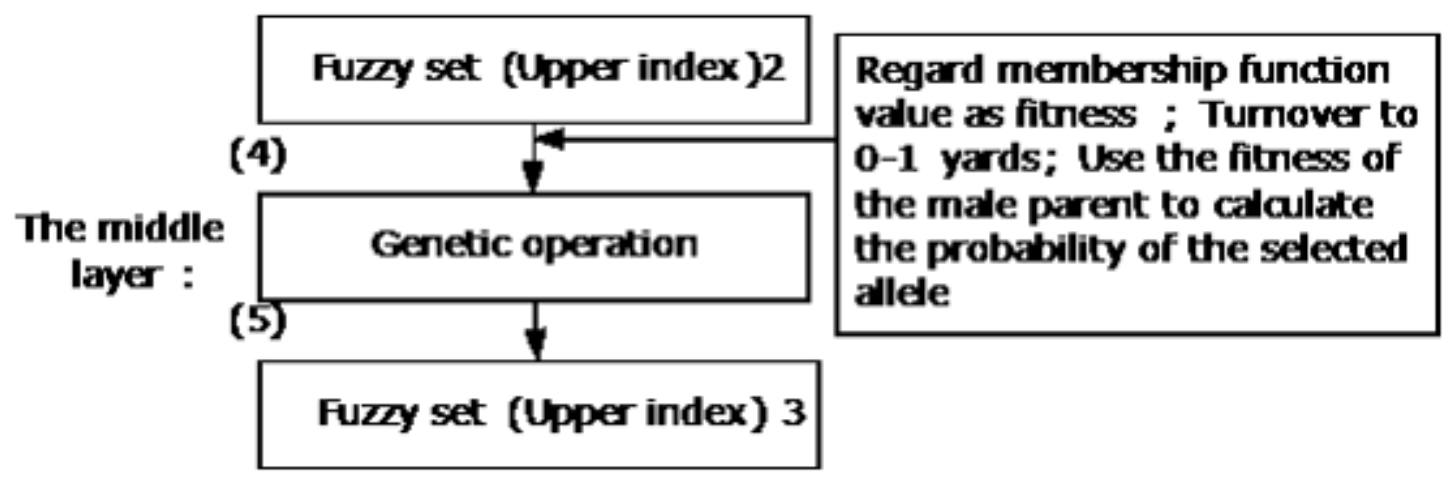

Figure 4. Computing the middle index value of FLI-GA model

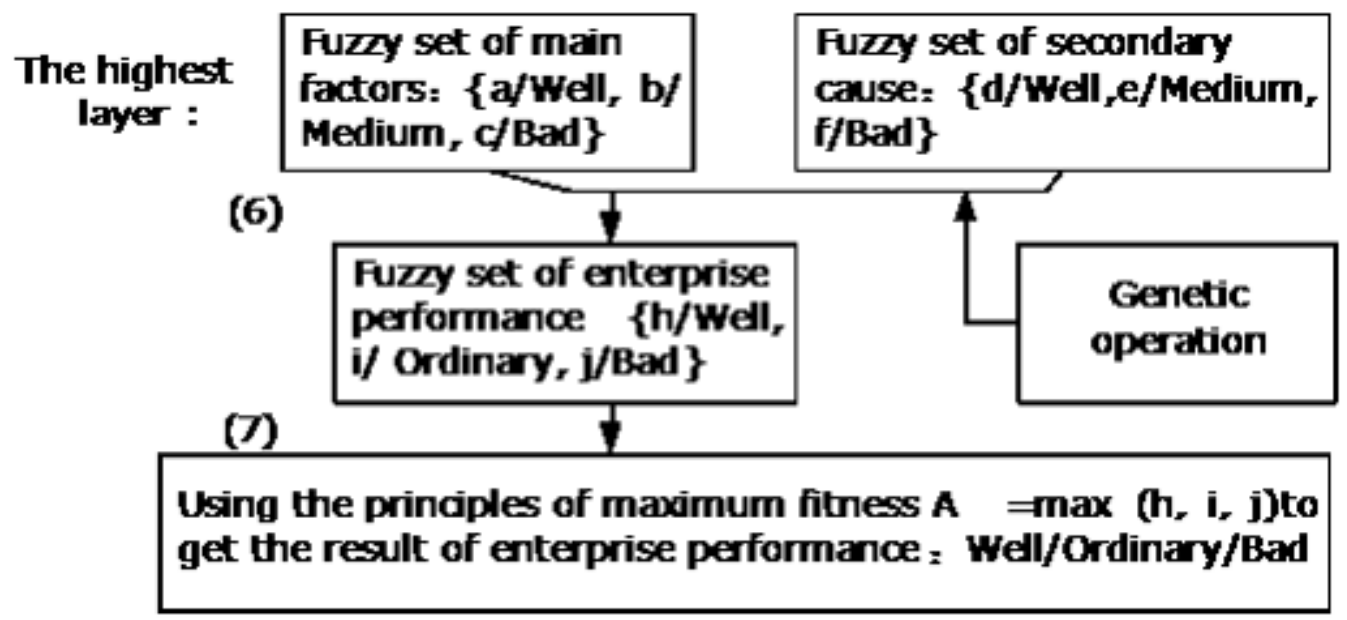

Figure 5. Computing the bottom index value of top tier 


\section{An Illustrative Example}

\subsection{Description of the example}

For the purpose of doing empirical research on enterprise performance evaluation, we have chosen an industrial automatic disciplinary enterprise $Z$ as an example to study. $Z$ Corporation is a developing enterprise and has the typical characteristics of enterprise performance evaluation indexes. Enterprise $Z$ was set up by relying on Industrial Automation National Engineering Research Center and Industrial Control Technology State Key Laboratory. This enterprise's main businesses are chemical, oil refining, petrochemical, metallurgy, electric power, light industry, textiles, building materials, pharmaceutical, and biochemical. It also makes use of advanced control strategies in industrial automation and control hardware platforms, as well as integrated automation control technology's research and production.

\subsection{The Input data of two models}

Enterprise $Z$ regularly invites experts to assess its performance, and each expert scores the indexes back-to-back to ensure fairness and justice. We selected the experts' scores of this enterprise in 2010 and 2011, as are shown in Table 2.

\begin{tabular}{|l|c|c|c|c|c|}
\hline \multicolumn{1}{|c|}{ Index } & $\begin{array}{c}\text { Expert 1 } \\
(\mathbf{2 0 1 0 / 2 0 1 1 )}\end{array}$ & $\begin{array}{c}\text { Expert 2 } \\
(\mathbf{2 0 1 0 / 2 0 1 1 )}\end{array}$ & $\begin{array}{c}\text { Expert 3 } \\
(\mathbf{2 0 1 0 / 2 0 1 1 )}\end{array}$ & $\begin{array}{c}\text { Expert 4 } \\
(\mathbf{2 0 1 0 / 2 0 1 1 )}\end{array}$ & $\begin{array}{c}\text { Expert 5 } \\
\mathbf{( 2 0 1 0 / 2 0 1 1 )}\end{array}$ \\
\hline $\begin{array}{l}\text { Current assets } \\
\text { turnover situation }\end{array}$ & $2.5 / 1.8$ & $1.5 / 2.4$ & $3.2 / 2.3$ & $4.0 / 2.6$ & $3.8 / 1.9$ \\
\hline $\begin{array}{l}\text { Total assets turnover } \\
\text { situation }\end{array}$ & $1.8 / 3.3$ & $2.0 / 3.5$ & $3.0 / 3.8$ & $3.2 / 3.6$ & $4.0 / 3.8$ \\
\hline Marketing capabilities & $3.8 / 4.0$ & $3.4 / 4.4$ & $4.1 / 4.5$ & $4.2 / 4.8$ & $4.5 / 4.8$ \\
\hline Revenue growth & $2.2 / 4.0$ & $1.8 / 4.2$ & $2.0 / 4.4$ & $2.3 / 4.3$ & $2.2 / 4.1$ \\
\hline Corporate culture & $3.0 / 4.2$ & $3.8 / 3.8$ & $3.8 / 4.4$ & $3.9 / 3.7$ & $3.0 / 3.9$ \\
\hline $\begin{array}{l}\text { Employee labor } \\
\text { enthusiasm }\end{array}$ & $2.8 / 1.8$ & $3.0 / 1.9$ & $3.2 / 2.2$ & $3.3 / 2.0$ & $2.7 / 2.1$ \\
\hline $\begin{array}{l}\text { Corporate sector } \\
\text { settings }\end{array}$ & $2.5 / 3.5$ & $2.8 / 3.3$ & $3.2 / 4.0$ & $3.0 / 3.7$ & $2.5 / 4.0$ \\
\hline
\end{tabular}

Table 2. Indexes scores in 2010/2011 (Enterprise Z)

Based on Table 2, we average the experts' score to get the value of indexes. The results are shown in Table 3. 


\begin{tabular}{|l|c|c|}
\hline \multicolumn{1}{|c|}{ Index } & Final score of 2011 & Final score of 2010 \\
\hline Current assets turnover situation & 2.2 & 3.0 \\
\hline Total assets turnover situation & 3.6 & 2.8 \\
\hline Marketing capabilities & 4.5 & 4.0 \\
\hline Revenue growth & 4.2 & 2.1 \\
\hline Corporate culture & 4.0 & 3.5 \\
\hline Employee labor enthusiasm & 2.0 & 3.0 \\
\hline Corporate sector settings & 3.7 & 2.8 \\
\hline
\end{tabular}

Table 3. The final indexes scores of enterprise $Z$

\subsection{Simulation of Fuzzy logic inference method}

This paper used the GUI tool of MATLAB to set and simulate the Fuzzy logic inference model. There are 4 main steps to simulate evaluation process by using fuzzy logic inference model:

- Firstly, build up the FIS system which contains the input and output variables, as well as the fuzzy rules;

- Edit the input and output membership function;

- Construct the inference rules;

- Get the reasoning results of the FIS system.

The evaluation results will be shown in next section. Because reasoning results of the fuzzy reasoning system come from the membership values which are selected from rule bases, or from maximizing/ minimizing operation, the final solution of fuzzy results become awfully close. Besides, whether the reasoning rule is good or bad will also significantly influence the final results. So it is necessary to add GA to improve the results.

\subsection{Simulation of FLI-GA model}

FLI-GA model's core idea is that when calculating the high-level indexes and target index, genetic algorithm is brought in to achieve the goals: encode options, cross, restructuring. The benefit of this method is that the numerical characteristics of the male parent can be kept and the best offspring can be selected out. This simulation model uses mid-level indexes output data from Chapter 4.3 as the genetic manipulation's male parent population. We programmed the program according to the calculation process from Chapter 3.2.

Matlab Genetic Algorithm Toolbox (GA)'s selection and crossover operators are given as an optional function, and problems which are solute by GA toolbox mainly belong to complete optimization and pattern search, so using Genetic Algorithm Toolbox is not entirely fit the 
simulation idea for this article. This article uses Matlab to compile custom simulation functions and processes to achieve the application of FLI-GA model. The detailed procedures are not discussed here, as well as detailed programs.

The simulation process follows the steps listed below:

- Offspring generated algorithm is defined as function geneSub (A1, $A 2)$, in which the parameters $\mathrm{A} 1$ and $\mathrm{A} 2$ are membership values from two male parents' comment, and the return value of such function is subPop.

- Function geneCal regards the male parent's seed (the seed is a $1 * 2$ matrix which contains two paternal chromosome information) as the entrance parameters; return values which named as geneRs is an "excellent" offspring chromosome. The so-called "excellent" means that the value the offspring geneRs maintains two basic information of the male parent, and geneRs has a better computational properties than the male parent, so in the merit-based process, since the $0-1$ cross-code operation generated two offspring whose values beyond their two parent, the two offspring will be discarded. Selected two offspring from the generated 500 offspring, which maintain the paternal characteristics (not beyond the paternal range), and are closest to their male parent values (that means this process narrow the range of the male parent values).

- The function geneLoop regards initial seed as its paternal import parameters, and returns the final optimal monomer fiRs. This function will doing iterative operation of multiple generations, and generates two "excellent" offspring by the male parent seed. Then these two offspring will generate "excellent" offspring. So we made circulative iteration for genetic manipulation until the last two offspring converge to a stable numerical. This is the signal of terminating the iteration operation. While in practical, except for the condition that iterative termination results converge to a particular point, there are also situations that the generated offspring from a generation back cannot be convergent but stop at two constant values. There will be no more optimizing offspring. This phenomenon shows that the genetic manipulation has been difficult to produce better offspring, and therefore it needs to use the maximum operation to select the best degree of membership.

- Function doFuzzy uses two index scores from low-level to be the entrance parameters. It transfers different genetic manipulation algorithms to get membership functions rsArr and index score rsScore of upper layer. 


\subsection{Comparison and Analyses}

Simulation result based on Fuzzy logic inference model shows: Enterprise Z's performance evaluation score of 2010 and 2011 are both 2.5, which belong to the "normal" degree at the semantic level. This result reflects that the performance of enterprise $Z$ is in the medium level. By contrast, the simulation result of FLI-GA model respectively gives two new scores 2.5107 and 2.5172. Table 4 shows the comparison of the two models.

\begin{tabular}{|l|c|c|}
\hline \multicolumn{1}{|c|}{ Model } & Performance of 2010 & Performance of 2011 \\
\hline $\begin{array}{l}\text { Fuzzy logic inference } \\
\text { model }\end{array}$ & 2.5 & 2.5 \\
\hline FLI-GA model & 2.5107 & 2.5172 \\
\hline
\end{tabular}

Table 4. Simulation results comparison between two models

Obviously, the results get from two models are nearly same. This means that the FLI-GA model keeps the basic result of Fuzzy logic inference model. At the same time, it also reveals that enterprise $Z$ remained the general level of performance. A closer examination reveals that FLIGA model is much more accurate. This phenomenon is not only shown in the results, but also been well represented in the simulation model's operation process.

Although we obtained similar results, during the FLI-GA model's genetic iteration process, the offspring produced by two male parents often convergence with a certain encoding monomer, or stay in two fixed values. This means that with the iteration process of the genetic manipulation, good genes of male parents have obtained the best choice. This "convergence" and "stay" phenomenon can be considered as fuzzy reasoning rules, namely, the fuzzy reasoning rules are instead by genetic manipulation of the genetic algorithm. These two models obtained similar results, but the reasoning results of the genetic algorithm are more stable. Compared with Fuzzy logic inference model's subjective and experiential reasoning rule, genetic manipulation is more objective and convincing.

In summary, two models can both effectively reflect the enterprise performance of the real situation, and the FLI-GA model's computing process is more stable and accurate.

\section{Conclusions}

In this paper, we firstly analyzed the existing Fuzzy logic inference method. Through the analysis, we find that this method has some defects and needs to be improved. Then we brought genetic algorithm into enterprise performance evaluation model on the basis of the improvement of original index system and rules library. This method can preferably solve a problem when index data are difficult to quantify in performance evaluation. The introduction 
of genetic algorithm is mainly used to determine an optimal evaluation standard. In addition, different theories play different roles in this evaluation model. We use genetic algorithm to refine inference rules and memberships of fuzzy reasoning method. By using genetic algorithm, the inference rules and memberships become more convincing. The results of simulations indicate that FLI-GA model is objective and accurate.

Though we have achieved some positive results, there are still some shortcomings in our research that need to be improved:

- The rule base needs to be improved continuously. With the development of society, the status and connotation of enterprise performance evaluation indexes are changing, so more perfect index system should be put forward. Besides, rule base of this research is static, while practical applications need dynamic rule base, therefore, we should try to establish dynamic rule base in further studies. Moreover, the convergence properties of genetic algorithms can be used on the previous studies to summarize and temper and this will make the rule base more objective and accurate.

- In FLI-GA model simulation, we haven't taken gene mutation into account. For the gene mutation is widespread in nature, and to make the model more reasonable, we should take gene mutation into consideration.

\section{Acknowledgments}

This work is supported by Beijing Natural Science Foundation (Grant No. 4112044), a major program of the National Social Science Fund of China 13\&ZD026 and Research Fund for the Doctoral Program of Higher Education of China (Grant No. 20120009110009).

\section{References}

Bi, X.Q., \& Zhang, F.F. (2010, July). The performance evaluation of enterprise knowledge management based on AHP. Proceedings of 2010 IEEE International Conference on Advanced Management Science, 2, 543-547. http://dx.doi.org/10.1109/ICAMS.2010.5552981

Golam, K., \& Akhtar, M.H.A. (2011). Evaluation of customer oriented success factors in mobile commerce using fuzzy AHP. Journal of Industrial Engineering and Management, 4(2), 361-386. http://dx.doi.org/10.3926/jiem.2011.v4n2.p361-386

Iraj, M., Mahyar, M., \& Fereydoun, A. (2011). Designing customer-oriented catalogs in e-CRM using an effective self-adaptive genetic algorithm. Expert Systems with Applications, 38(1), 631-639. http://dx.doi.org/10.1016/j.eswa.2010.07.013

Lou, W.G., \& Kuang, L.K. (2010, Nov.). Comprehensive Evaluation of Competitiveness of Listed 
Companies Using Artificial Neural Network. 2010 International Conference on E-Product EService and E-Entertainment, ICEEE2010, Henan, China, 7-9 Nov., 2010.

http://dx.doi.org/10.1109/ICEEE.2010.5660325

Ma, S.N., He, J.S., \& Shuai, X.B. (2011). Application of Fuzzy Comprehensive Evaluation Method in Trust Quantification. International Journal of Computational Intelligence Systems, 4(5), 768-776. http://dx.doi.org/10.1080/18756891.2011.9727828

Patrick, K., Douglas, R., \& Fabio, C. (2010). Diarization of Telephone Conversations Using Factor Analysis. Journal of Selected Topics in Signal Processing, 4(6), 1059-1070. http://dx.doi.org/10.1109/JSTSP.2010.2081790

Pond, S.L.K., Posada, D., Gravenor, M.B., Woelk, C.H., \& Frost, S.D.W. (2006). Automated phylogenetic detection of recombination using a genetic algorithm. Molecular Biology and Evolution, 23(10), 1891-1901. http://dx.doi.org/10.1093/molbev/msl051

Qi, E.S., \& Sun, W.J. (2011). Research on the performance evaluation system of private enterprise based on AHP and PCA. 2011 IEEE 18Th International Conference on Industrial Engineering and Engineering Management (IE\&EM), Part 1, 201-204, Changchun, China, 3-5 Sep., 2011. http://dx.doi.org/10.1109/ICIEEM.2011.6035139

Rustum, M., Omar, B., \& Emad, A. (2009). A fuzzy inference model for short-term load forecasting. Energy Policy, 37(4), 1239-1248. http://dx.doi.org/10.1016/j.enpol.2008.10.051

Saeed, R., Mehdi, G., \& Mostafa, J. (2012). Evaluation model of business intelligence for enterprise systems using fuzzy TOPSIS. Expert Systems with Applications, 39(3), 3764-3771. http://dx.doi.org/10.1016/j.eswa.2011.09.074

Yang, Z., Zhang, T., Wang, Y., Wang, W.W., \& Zhang, X.F. (2010, Nov.). Logistics enterprise performance evaluation based on entropy weight and grey correlation. 2010 International Conference on E-Product E-Service and E-Entertainment, ICEEE2010, Henan, China, 7-9 Nov., 2010. http://dx.doi.org/10.1109/ICEEE.2010.5660355

Zhang, C.Q., Xie, P., \& Cao, M.M. (2011). Research on environmental performance evaluation index system of Power Generation Enterprise. Proceedings of the 6th International Forum on Strategic Technology, IFOST 2011, 632-635, China, 22-24 Aug., 2011.

http://dx.doi.org/10.1109/IFOST.2011.6021101

Zhang, J.S., \& Tan, W. (2012). Research on the Performance Evaluation of Logistics Enterprise Based on the Analytic Hierarchy Process. Energy Procedia, 14, 1618-1623.

http://dx.doi.org/10.1016/j.egypro.2011.12.1142

Journal of Industrial Engineering and Management, 2014 (www.jiem.org)

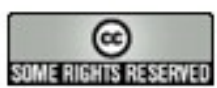

Article's contents are provided on a Attribution-Non Commercial 3.0 Creative commons license. Readers are allowed to copy, distribute and communicate article's contents, provided the author's and Journal of Industrial Engineering and Management's names are included. It must not be used for commercial purposes. To see the complete license contents, please visit http://creativecommons.org/licenses/by-nc/3.0/. 